\title{
Analysis of all subunits, SDHA, SDHB, SDHC, SDHD, of the succinate dehydrogenase complex in KIT/PDGFRA wild-type GIST
}

\author{
Maria A Pantaleo ${ }^{\star 1}$, Annalisa Astolfi ${ }^{2}$, Milena Urbini ${ }^{2}$, Margherita Nannini ${ }^{1}$, Paola Paterini ${ }^{2}$, Valentina Indio ${ }^{2}$, \\ Maristella Saponara ${ }^{1}$, Serena Formica ${ }^{2}$, Claudio Ceccarelli ${ }^{3}$, Rita Casadio ${ }^{4}$, Giulio Rossi ${ }^{5}$, Federica Bertolini ${ }^{5}$, \\ Donatella Santini $^{3}$, Maria G Pirini ${ }^{3}$, Michelangelo Fiorentino ${ }^{6}$, Umberto Basso ${ }^{7}$ and \\ Guido Biasco $^{1,2}$ on behalf of GIST Study Group
}

\begin{abstract}
Mutations of genes encoding the subunits of the succinate dehydrogenase (SDH) complex were described in KIT/PDGFRA wild-type GIST separately in different reports. In this study, we simultaneously sequenced the genome of all subunits, SDHA, SDHB, SDHC, and SDHD in a larger series of KIT/PDGFRA wild-type GIST in order to evaluate the frequency of the mutations and explore their biological role. SDHA, SDHB, SDHC, and SDHD were sequenced on the available samples obtained from 34 KIT/PDGFRA wild-type GISTs. Of these, in 10 cases, both tumor and peripheral blood (PB) were available, in 19 cases only tumor, and in 5 cases only PB. Overall, 9 of the 34 patients with KIT/PDGFRA wild-type GIST carried mutations in one of the four subunits of the SDH complex (six patients in SDHA, two in SDHB, one in SDHC). WB and immunohistochemistry analysis showed that patients with KIT/PDGFRA wild-type GIST who harbored SDHA mutations exhibited a significant downregulation of both SDHA and SDHB protein expression, with respect to the other GIST lacking SDH mutations and to KIT/PDGFRA-mutated GIST. Clinically, four out of six patients with SDHA mutations presented with metastatic disease at diagnosis with a very slow, indolent course. Patients with KIT/PDGFRA wild-type GIST may harbor germline and/or de novo mutations of SDH complex with prevalence for mutations within SDHA, which is associated with a downregulation of SDHA and SDHB protein expression. The presence of germline mutations may suggest that these patients should be followed up for the risk of development of other cancers. European Journal of Human Genetics (2014) 22, 32-39; doi:10.1038/ejhg.2013.80; published online 24 April 2013
\end{abstract}

Keywords: SDHA; SDHB; SDHC; SDHD; wild-type GIST; gastrointestinal stromal tumors

\section{INTRODUCTION}

Approximately $85 \%$ of gastrointestinal stromal tumors (GIST) in adult patients harbor gain-of-function mutations in either the KIT gene or the platelet-derived growth factor receptor-alfa (PDGFRA) gene that lead to tumor development, resulting in the constitutive ligand-independent activation of the receptor tyrosine kinases and their downstream signaling pathways. ${ }^{1}$ Approximately $10 \%$ of GISTs in adult patients and notably, approximately $85 \%$ of GIST in children do not harbor a mutation in either gene (defined as KIT/PDGFRA wild type) and are often associated with a cancer syndrome. ${ }^{2,3}$ In KIT/PDGFRA wild-type GIST, activating mutations in BRAF have been reported. ${ }^{4}$ Also, the presence of mutations on SDHB and SDHC, (which encode subunits B and C, respectively, of succinate dehydrogenase (SDH) or complex II) and, more recently, mutations on SDHA have been described separately in different reports. ${ }^{5-12}$ Currently, the simultaneous sequencing of all subunits, A, B, C, and $\mathrm{D}$ of SDH complex has not been reported in a large series of clinically non-syndromic GIST that are KIT- and PDGFRA-mutation negative.
The aims of this work were to study the frequency of SDH genes mutations evaluating simultaneously all SDH complex subunits in KIT/PDGFRA wild-type GIST, to explore their biological role, and ultimately to discuss specific features that would potentially be interesting from a clinical point of view.

\section{MATERIALS AND METHODS}

Patients and tumors

Among 358 patients with GIST analyzed for KIT (exons 9, 11, 13, and 17) and PDGFRA (exons 12, 14, and 18) mutations, 34 GISTs were found to be KIT/ PDGFRA wild type. We focused the study of SDH complex on this population of 34 patients, including the 2 patients already studied. ${ }^{6}$ We sequenced SDHA, SDHB, SDHC, and SDHD on both tumor (T) and peripheral blood (PB) DNA in 10 patients and only on T DNA in 19 patients. In five patients, the DNA extracted from the formalin-fixed paraffin-embedded (FFPE) slides available at our center was insufficient or too degraded for SDH mutational analysis, but for these patients the PB was collected. We decided to study also these five cases with only $\mathrm{PB}$ available in order to explore the presence of germline mutations on PB DNA since germline and somatic loss-of-function mutations in SDHA were previously described. ${ }^{6}$ Patient and tumor characteristics are

${ }^{1}$ Department of Specialized, Experimental and Diagnostic Medicine, Sant'Orsola-Malpighi Hospital, University of Bologna, Bologna, Italy; 2“Giorgio Prodi’ Cancer Research Center, University of Bologna, Bologna, Italy; ${ }^{3}$ Pathology Unit, Sant'Orsola-Malpighi Hospital, University of Bologna, Bologna, Italy; ${ }^{4}$ Biocomputing Group, Department of Biology, University of Bologna, Bologna, Italy; ${ }^{5}$ Section of Pathologic Anatomy and Division of Oncology, Azienda Ospedaliero-Universitaria Policlinico di Modena, Modena, Italy; ${ }^{6}$ Laboratorio di Patologia Molecolare Oncologica e dei Trapianti—Pathology Unit, Sant'Orsola-Malpighi Hospital, University of Bologna, Bologna, Italy; ${ }^{7}$ Oncologia Medica 1, Istituto Oncologico Veneto IOV I.R.C.C.S., Padova, Italy

${ }^{*}$ Correspondence: MA Pantaleo, Department of Specialized, Experimental and Diagnostic Medicine, S. Orsola-Malpighi Hospital, University of Bologna, 40138 Bologna, Italy. Tel: + 3951 6364078; Fax: + 3951 6364037; E-mail: maria.pantaleo@unibo.it

Received 13 November 2012; revised 31 January 2013; accepted 24 March 2013; published online 24 April 2013 
listed in Supplementary Table S1. The family and personal history of these patients were assessed at the time of the first clinical visit and were confirmed after the present results. The screening for other tumors, paraganglioma, or pheochromocytoma was done by CT scan every 6 months during the follow-up for GISTs.

\section{Mutational analysis of SDH subunits}

The exons of the four subunits of SDH complex (SDHA exons 1-15, SDHB exons $1-8$, SDHC exons $1-6$, and SDHD exons 1-4) were sequenced on tumor and/or PB of patients with KIT/PDGFRA wild-type GIST using the Sanger sequencing method on ABI 3730 Genetic Analyzer (Applied Biosystems, Monza, Italy). DNA was extracted from tumor specimens by the QIAmp DNA Mini or Micro kit (Qiagen, Milan, Italy) in accordance with manufacturer's directions. Primer pairs, designed with Primer Express 3.0 Software (Applied Biosystems), were specific to amplify exons and the flanking intronic regions but not SDHA pseudogenes located in chromosomes 3 and 5. Primer sequences are listed in supporting information Supplementary Table S2. PCR products were purified with the Qiaquick PCR purification kit (Qiagen) and sequenced on both strands using the Big Dye Terminator v1.1 Cycle Sequencing kit (Applied Biosystems).

\section{Immunohistochemistry (IHC) of SDHA and SDHB}

SDHA and SDHB were evaluated in 20 out of 34 patients with KIT/PDGFRA wild-type GIST where the FFPE tissue was available. Of these 20 cases, 14 did not have SDH complex mutations, 4 had SDHA mutations, 1 had SDHB mutation, and 1 had SDHC mutation. Moreover, 10 patients with KIT/ PDGFRA-mutant GIST (three mutated in KIT exon 9, four mutated in KIT exon 11, and three mutated in PDGFRA exon 18) were evaluated. IHC was performed on 4- $\mu \mathrm{m}$ sections of FFPE GIST tumor samples. Mouse monoclonal anti-SDHA (ab14715, Abcam, Cambridge, UK, 1:2500) and rabbit polyclonal anti-SDHB (HPA002868, Sigma-Aldrich, St Louis, MO, USA, 1:800) antibodies were used. The sections were deparaffinized, rehydrated, and subjected to the appropriate antigen retrieval treatment (for SDHA: microwave heating in TRIS-EDTA buffer, $\mathrm{pH} 9.0$ at $100^{\circ} \mathrm{C}$ for $20 \mathrm{~min}$; SDHB: microwave heating in citrate buffer $\mathrm{pH} 6.0$ at $100^{\circ} \mathrm{C}$ for $40 \mathrm{~min}$ ). After cooling at room temperature, the activity of endogenous peroxidises was inhibited using methanol/ $\mathrm{H}_{2} \mathrm{O}_{2}$ $(0.5 \% \mathrm{v} / \mathrm{v})$ for $20 \mathrm{~min}$. The sections were then washed in phosphate-buffered saline (PBS, pH 7.2-7.4) and incubated with the specific primary antibody overnight at room temperature. After that, the sections were washed in PBS and treated using the Novolink Polymer Detection System (Novocastra, Newcastle upon Tyne, UK) according to the manufacturer's instructions. In the negative control, primary antibodies were omitted. Human seminal vesicles (for SDHA) and liver tissues (for SDHB) were used as positive controls. These tissues showed strong granular staining in the cytoplasm and mitochondria with both of the antibodies.

Protein extraction and western blot analysis of SDHA and SDHB Protein expression of SDHA and SDHB was evaluated on 8 KIT/PFGFRA wild type and five KIT/PDGFRA mutant GISTs for which fresh-frozen tissues were available. Tissue was disrupted in RIPA buffer (Sigma-Aldrich) supplemented with protease inhibitors ( $1 \mathrm{~mm}$ PMSF, $10 \mu \mathrm{g} / \mathrm{ml}$ aprotinin, $10 \mu \mathrm{g} / \mathrm{ml}$ leupeptin, $1 \mathrm{~mm}$ orthovanadate sodium salt), and lysed for $1 \mathrm{~h}$ with gentle agitation at $4{ }^{\circ} \mathrm{C}$. Lysates were centrifuged at $13000 \times g$ for $15 \mathrm{~min}$ at $4{ }^{\circ} \mathrm{C}$ and supernatants were stored at $-80^{\circ} \mathrm{C}$. Protein concentrations were determined with the BCA protein assay (Pierce, Rockford, IL, USA). Thirty micrograms of protein was resolved on a 15\% SDS-PAGE gel and transferred onto polyvinylidene difluoride membranes. Nonspecific binding sites were blocked by incubation in blocking buffer (PBS containing 0.1\% Tween-20 with 5\% w/v BSA) for $1 \mathrm{~h}$ at room temperature. Membranes were incubated overnight at $4{ }^{\circ} \mathrm{C}$, with the following primary antibodies: mouse monoclonal SDHA antibody (ab14715, Abcam, 1:10.000), rabbit monoclonal SDHB antibody (HPA002868, SigmaAldrich, 1:500), and rabbit polyclonal $\beta$-Actin antibody (sc-8432, Santa Cruz Biotechnology, Santa Cruz, CA, USA, 1:500). Then membranes were washed and incubated with peroxidase conjugate secondary antibodies for $1 \mathrm{~h}$ at room temperature. Antigens were revealed using Enhanced Chemiluminescence Reaction (ECL Advance, Amersham Pharmacia Biotech, Les Ulis, France).

\section{Bioinformatic analysis}

Bioinformatic analysis was necessary to predict the effect of the detected mutations in the targeted genomic sequences, specifically focusing on nonsynonymous mutations (considering dbSNP and 1000 genomes databases) promoting an amino-acid substitution in the corresponding translated protein. In such cases, the effect of the variation on the protein chain was predicted with different tools such as: (I) 'SNP\&GO', a predictor of human diseaserelated mutations in proteins that considers information from protein sequence, evolutionary information, and gene ontology terms; ${ }^{13}$ (II) 'SIFT', a predictor based on the degree of conservation of amino-acid residues in sequence alignments derived from closely related sequences, collected through PSI-BLAST; ${ }^{14}$ (III) 'PolyPhen-2', a tool that predicts possible impact of an amino-acid substitution on the structure and function of a human protein using straightforward physical and comparative considerations; ${ }^{15}$ (IV) 'I-Mutant2.0', a neural network-based web server for the automatic prediction of protein stability changes upon single-site mutations, ${ }^{16}$ and (V) 'MutPred', a web application tool developed to classify an amino-acid substitution in humans as disease-associated or neutral. ${ }^{17}$

All of these methods are among the state-of-the-art approaches in computing whether a variation is disease associated or not (I-III, V), and whether a variation is affecting the protein stability (IV). We also used a predictor of transmembrane helix domains to locate the variation in SDHC. ${ }^{18}$

Variations were mapped on the human SDH, after modeling the protein on its homologous pig counterpart as previously described. ${ }^{6}$ The human SDH model was plotted with UCSF Chimera software ${ }^{19}$ that was adopted also to calculate the distance between the mutated position and the flavine adenine dinucleotide (FAD) binding site. Moreover, the program 'Dictionary Of Protein Secondary Structure' (DSSP) was employed in order to compute the solvent accessibility for each mutated residues. ${ }^{20}$ The solvent exposure calculation was performed separately considering the variation in the assembled SDH complex and in the single subunit.

Schematic diagrams of interactions within the proteins were also computed with 'LIGPLOT;' the tool allows comparison of the arrangement of local environment in wild type with respect to mutated protein. ${ }^{21}$

Two different splicing site predictors, 'ASSP-Alternative Splicing Site Predictor' $^{22}$ and NetGene2 'server,' ${ }^{23}$ were used to predict the effect of noncoding mutations occurring in the exon-intron proximity.

\section{RESULTS}

\section{Mutational analysis of SDH subunits}

SDHA, SDHB, SDHC, and SDHD were sequenced on both T and PB in 10 cases, only on $\mathrm{T}$ in 19 cases, and only in PB in 5 cases; in 1 case, there was enough material only to perform SDHA sequencing. The mutations found are reportedin Table 1, and chromatograms of individual mutations are shown in Figure 1. With regard to SDHA, six patients harbored in the tumor sample either homozygous or compound heterozygous mutations for nine mutations globally in this gene. In addition to those previously identified in the patients GIST_07 and GIST_10, six new SDHA mutations were found in another four samples. In particular, one case (GIST_24) harbored a c.1046_1047delTG mutation in exon 8 in a tumor sample that leads to a premature stop codon in the protein (p.L349R fs ${ }^{\star} 11$ ). One case (GIST_145) harbored two heterozygous missense mutations in exons 5 and 13, R171C present only in tumor sample and R589Q both in tumor and PB. The other case (GIST_214) harbored G419R and E564K heterozygous missense mutations in exons 9 and 13, respectively, in the tumor sample. The last case (GIST_234) harbored a c.457-3_457-1 delCAG, heterozygous mutation in PB and homozygous in the tumor. With regard to SDHB, a heterozygous mutation (c.301delT) was found in exon 4 in one PB sample (GIST_311) that led to a stop codon at amino-acid position 103; for this case, tumor tissue was not available, while another case (GIST_270) harbored a c. $423+20 \mathrm{~T}>\mathrm{A}$ in exon 4-intron boundary in the tumor sample that leads to presumed damage during the process of RNA maturation. 
Table 1 SDH mutations identified in KIT/PDGFRA wild-type GIST and characteristics of patients and tumors

SDH mutational status

\begin{tabular}{|c|c|c|c|c|c|c|c|c|c|c|}
\hline$I D$ & Gender & Age & Site & Multifocal & $\begin{array}{c}\text { Histological } \\
\text { type }\end{array}$ & $\begin{array}{l}\text { Disease status } \\
\text { at diagnosis }\end{array}$ & Subunits & Variation & Tumor & $P B$ \\
\hline GIST_07a & $\mathrm{F}$ & 28 & Stomach & Yes & Mixed & $\begin{array}{l}\text { Metastatic (liver, } \\
\text { lymph nodes, lung) }\end{array}$ & SDHA (Exon 9) & $\begin{array}{l}\text { c. } 1151 \mathrm{C}>\mathrm{G} \\
\text { p.S384X }\end{array}$ & Homozygous & Heterozygous \\
\hline \multirow[t]{2}{*}{ GIST_10a } & M & 30 & Stomach & No & Mixed & $\begin{array}{l}\text { Metastatic (liver, } \\
\text { lymph nodes) }\end{array}$ & SDHA (Exon 2) & $\begin{array}{l}\text { c. } 91 \mathrm{C}>\mathrm{T} \\
\text { p.R31X }\end{array}$ & Heterozygous & Heterozygous \\
\hline & & & & & & & SDHA (Exon 13) & $\begin{array}{l}\text { c. } 1765 \mathrm{C}>\mathrm{T} \\
\text { p.R589W }\end{array}$ & heterozygous & absent \\
\hline GIST_24 & $\mathrm{F}$ & 18 & Stomach & NA & NA & Liver & SDHA (Exon 8) & $\begin{array}{l}\text { c.1046_1047delTG } \\
\text { p.L349R fs*11 }\end{array}$ & Heterozygous & NA \\
\hline \multirow[t]{2}{*}{ GIST_145 } & $\mathrm{F}$ & 39 & Stomach & No & Epithelioid & $\begin{array}{l}\text { Metastatic } \\
\text { (liver, lymph nodes) }\end{array}$ & SDHA (Exon 5) & $\begin{array}{l}\text { c. } 511 \mathrm{C}>\mathrm{T} \\
\text { p.R171C }\end{array}$ & Heterozygous & Absent \\
\hline & & & & & & & SDHA (Exon 13) & $\begin{array}{l}\text { c. } 1766 \mathrm{G}>\mathrm{A} \\
\text { p.R589Q }\end{array}$ & Heterozygous & Heterozygous \\
\hline \multirow[t]{2}{*}{ GIST_214 } & $\mathrm{F}$ & 17 & Stomach & Yes & Mixed & Not metastatic & SDHA (Exon 9) & $\begin{array}{l}\text { c. } 1255 \mathrm{G}>\mathrm{A} \\
\text { p.G419R }\end{array}$ & Heterozygous & NA \\
\hline & & & & & & & SDHA (Exon 13) & $\begin{array}{l}\text { c. } 1690 \mathrm{G}>\mathrm{A} \\
\text { p.E564K }\end{array}$ & heterozygous & NA \\
\hline GIST_234 & $\mathrm{F}$ & 37 & Stomach & No & Epithelioid & Not metastatic & SDHA (Exon 5) & $\begin{array}{l}\text { c.457-3_457-1 } \\
\text { delCAG }\end{array}$ & Homozygous & Heterozygous \\
\hline GIST_270 & $M$ & 77 & Colon & No & Mixed & Not metastatic & SDHB (intron 4) & c. $423+20 \mathrm{~T}>\mathrm{A}$ & Heterozygous & NA \\
\hline GIST_281 & M & 67 & Duodenum & No & Spindle & Not metastatic & SDHC (Exon 6) & $\begin{array}{l}\text { c. } 455 G>C \\
\text { p.E144Q }\end{array}$ & Heterozygous & NA \\
\hline GIST_311 & $M$ & 41 & Stomach & No & NA & Metastatic & SDHB (Exon 4) & $\begin{array}{l}\text { c.301delT } \\
\text { p.C101V fs*3 }\end{array}$ & NA & Heterozygous \\
\hline
\end{tabular}

Abbreviations: NA, not available; PB, peripheral blood.

aThe mutations carried by these patients were already reported [6]

a GIST_145 PB DNA
SDHA exon 5

\begin{tabular}{ccccccccc}
$C$ & $A$ & $G$ & $C$ & $G$ & $T$ & $G$ & $C$ & $A$ \\
\hline $\mathbf{d}$ & $T$ & $J$ & $\mathbf{D}$ & $J$ & $A$ & $J$ & $\mathbf{D}$ & $T$
\end{tabular}

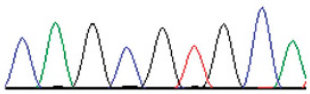

GIST_145 tumor DNA SDHA exon 5 c. $511 \mathrm{C}>\mathrm{T}$ \begin{tabular}{ccccccccc}
$C$ & $A$ & $G$ & $Y$ & $G$ & $T$ & $G$ & $C$ & $A$ \\
\hline J & $T$ & $J$ & $G$ & $J$ & $A$ & $J$ & A & $T$
\end{tabular}

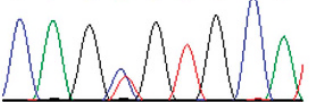

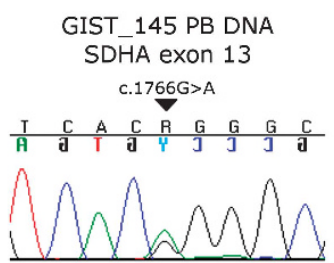

c

GIST_234 PB DNA SDHA exon 5

c.457-3_457-1 delCAG

c. $1690 \mathrm{G}>\mathrm{A}$
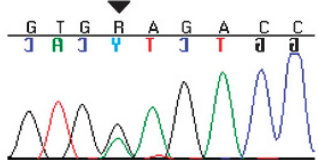
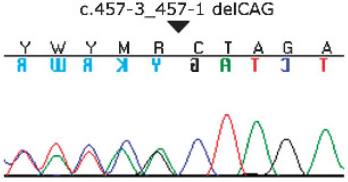
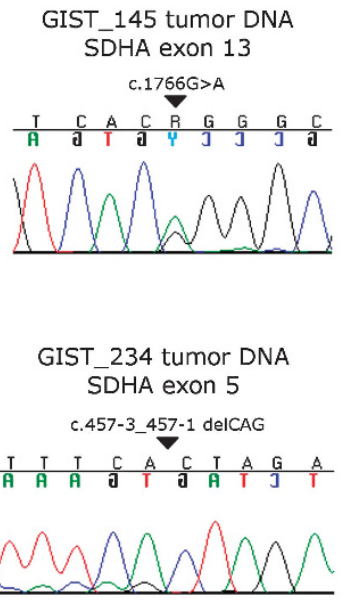
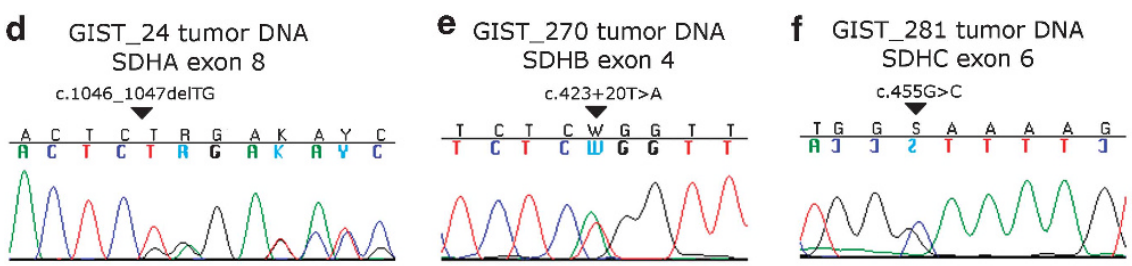

g GIST_311PB DNA

SDHB exon 4

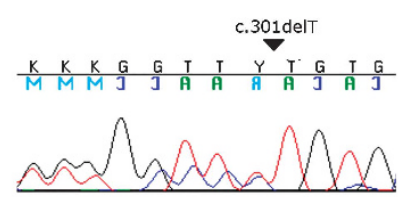

Figure 1 Chromatogram showing all the SDH mutations found in 34 KIT/PDGFRA wild-type GIST tumors or PB samples. (a) SDHA mutations in exon 5 and 13 carried by GIST_145; (b) SDHA mutations in exon 9 and 13 carried by GIST_214; (c) GIST_234 deletion in exon 5 of SDHA; (d) SDHA deletion in exon 8 carried by GIST_24; (e) GIST_270 mutation in exon 4 of SDHB; (f) GIST_281 mutation in exon 6 of SDHC; (g) GIST_311 deletion in exon 4 of SDHB. 
Table 2 Prediction of missense mutation effect on SDH protein subunits

\begin{tabular}{|c|c|c|c|c|c|c|}
\hline Protein subunit & Mutation & SNPS\&GO prediction & SIFT prediction & PolyPhen-2 prediction & I-Mutant 2.0 prediction & MutPred prediction \\
\hline SDHA & p.R171C & Disease (9) & Damaging(0) & Damaging (1.0) & Decrease stability (3) & Probable deleterious (0.921) \\
\hline SDHA & p.R589Q & Disease (9) & Damaging(0) & Damaging (1.0) & Decrease stability (6) & Probable deleterious (0.947) \\
\hline SDHA & p.G419R & Disease (9) & Damaging(0) & Damaging (1.0) & Decrease stability (2) & Probable deleterious $(0.921)$ \\
\hline SDHA & p.E564K & Disease (5) & Damaging(0) & Damaging (1.0) & Decrease stability (8) & Probable deleterious $(0.724)$ \\
\hline SDHC(isoform2) & p.E144Q & Disease (4) & Damaging(0) & Benign (0.255) & Decrease stability (7) & Probable deleterious (0.820) \\
\hline
\end{tabular}

The predictions were tabulated with a short description of missense mutation effect and a reliability index or a prediction score according to the computational method. (I) SNP2\&GO:

'disease-related' or 'neutral' with a reliability index ranging from 0-10; (II) SIFT: a score ranging from 0 ('damaging') and 1 ('tolerated'); (III) PolyPhen-2: a score ranging from 0 ('benign') and

1 ('damaging'); (IV) I-Mutant3.0: 'increase stability' or 'decrease stability' with a reliability index ranging from 0 to 10 ; (V) MutPred: probability of deleterious mutation ranging from 0 (not

confident hypothesis) to 1 (very confident hypothesis).

Only one SDHC mutation (E144Q) in the isoform 2 was found in exon 6 in the tumor sample of one patient (GIST_281), while SDHD did not show any mutation in any of the 34 patients with wild-type GIST. Overall, 9 of the 34 patients with KIT/PDGFRA wild-type GIST (26.4\%) carried mutations in one of the four subunits of the SDH complex with a predominance of SDHA mutations on the other subunits (six patients with subunit A mutation, with respect to two SDHB and one SDHC mutations).

The coding non-synonymous mutations were predicted with five different computational tools. Remarkably, the data indicate that all the different tools, albeit based on different assumptions, indicate a high probability of protein damage for the different variations experimentally detected in the corresponding exons (Table 2). Mapping of the damaging variations on the protein subunits is shown in Figure 2. It appears that all of the variations (although detected in different patients) were found far from the protein active site (detailed distances are reported in the figure legend). We tested SDHA deletion c.457-3_457-1 delCAG that is located immediately upstream the exon 5 with two different splicing site predictors; both tools were in agreement in predicting an alternative splicing site when the three bases CAG were deleted. The possible splicing site predicted led to a change of exon phase with a consequent frame shift that introduced a premature stop codon.

\section{SDHA and SDHB protein expression}

We evaluated by IHC the expression of SDHA and SDHB in FFPE tumor samples (from 20 patients with KIT/PDGFRA wild-type GIST and 10 patients with GIST with KIT- or PDGFRA-activating mutations) and in fresh frozen tissue of 8 KIT/PDGFRA wild-type and 5 KIT/PDGFRA mutant GIST by western blotting. Western blot analysis showed that four patients with KIT/PDGFRA wild-type GIST who harbored mutations in SDHA showed a significant downregulation of both SDHA and SDHB proteins with regard to the other four KIT/ PDGFRA wild-type GIST lacking mutations in the SDH complex and to the KIT/PDGFRA mutant GISTs (Figure 3). Results of IHC analysis showed that patients with KIT or PDGFRA mutations exhibited a strong granular staining for SDHA and SDHB in the cytoplasm and mitochondria, regardless of the type of mutation. Among patients with KIT/PDGFRA wild-type GIST, those without SDH complex mutations showed a similar SDHA and SDHB expression compared with patients with KIT/PDGFRA mutations (Figure 4A and Table 3); patients mutated for SDHA showed a negative staining for both SDHA and SDHB proteins while patients with SDHB or SDHC mutation showed a similar strong staining for SDHA compared with mutated patients and a negative staining of SDHB antibody (Figure 4B and Table 3).

\section{Clinical features of patients with SDH mutations}

All patients with SDH mutations did not present with a personal history of paraganglioma or family history of paraganglioma and GIST. The clinical and tumor data of patients with SDH mutations are summarized in the Table 1. With regard to the clinical outcome, among patients with SDHA mutations, four had a metastatic disease at diagnosis. Two of them (GIST_07 and GIST_145) is receiving imatinib as first-line treatment and then experienced a prolonged period of disease stabilization under sunitinib treatment (22 and 20 months, respectively). They are currently receiving nilotinib and are experiencing a further period of disease stabilization (58 and 60 months, respectively). ${ }^{24}$ The third patient (GIST_07) underwent surgical removal of primary GIST, liver and lymph node metastases, and received imatinib in the adjuvant setting. All of these three patients with metastatic disease and SDHA mutations presented with a very long and slow clinical indolent course (more than 5 years from diagnosis). In last one metastatic case, the follow-up was not available. Among the two patients with localized disease, one underwent the resection of primary GIST classified as low risk of metastatic recurrence according to Miettinen's classification but experienced the development of new gastric GISTs 13 years after the first operation. No data were available on the follow-up treatment of new lesions. The last patient who underwent the resection of primary tumor was classified as low risk of recurrence according to Miettinen's classification and she did not receive imatinib treatment.

Regarding the patients with SDHB and SDHC mutations, in our series the clinical and tumor characteristics were very different for age, site, and disease presentations. In addition, the number of these patients was too small to support any conclusions from a clinical point of view.

\section{DISCUSSION}

In recent years, new molecular and clinical data in KIT/PDGFRA wild-type GIST (occurring in approximately 10-15\% in adults) have been accumulated suggesting that it is time to consider this small subtype of GIST as a 'family of disease' and not as a unique entity. In our series of adult and young adult patients with KIT/PDGFRA wildtype GIST, global mutations in SDH complex were found in about $26.4 \%$ of these patients with a prevalence for the subunit $\mathrm{A}$ in six patients, mutations in subunit SDHB in only two patients, and mutation in subunit SDHC in only one patient. In general, SDHA mutations were predominant. All of the SDHA-mutated cases for which PB was available (four out of six) showed the presence of one mutation in the germline suggesting the presence of genetic predisposition to develop the tumor; in the other cases, the mutations were identified only in the tumor or in PB because, unfortunately, the matched samples were not available. In particular, in two cases, the 


\begin{tabular}{|c|c|c|c|c|}
\hline \multicolumn{5}{|c|}{ Mitochondrial inter-membrane space } \\
\hline \multicolumn{5}{|l|}{ Mitochondrial inner membrane } \\
\hline \multicolumn{5}{|l|}{ Mithochondrial matrix } \\
\hline \multirow{2}{*}{\multicolumn{5}{|c|}{$\begin{array}{l}\text { GIST 214: } \\
\text { SDHA-E564K } \\
\text { SDHA-G419R } \\
\text { GIST 07: } \\
\text { SDHA-S384X } \\
\text { GIST_24: } \\
\text { SDHA-L349R fs } * 11\end{array}$}} \\
\hline & & & & \\
\hline Variation & Patient & $\begin{array}{l}\text { Solvent Accessibility } \\
\text { (SDH complex) }\end{array}$ & $\begin{array}{l}\text { Solvent Accessibility } \\
\text { (single subunit) }\end{array}$ & $\begin{array}{l}\text { Distance from FAD binding } \\
\text { site }(\hat{\Lambda})\end{array}$ \\
\hline SDHA R171C & GIST_145 & $56.6 \%$ & $56.6 \%$ & 23.3 \\
\hline SDHA R589Q & GIST_145 & $0.4 \%$ & $0.4 \%$ & 26.1 \\
\hline SDHA G419R & GIST 214 & $0 \%$ & $0 \%$ & 23.7 \\
\hline SDHA E564K & GIST_214 & $9.1 \%$ & $44.4 \%$ & 16.9 \\
\hline SDHA R31X' & GIST_10 & I & I & I \\
\hline SDHA R589W & GIST_10 & 1 & 1 & I \\
\hline SDHA S384Xำ & GIST 7 & $0.4 \%$ & $0.4 \%$ & 26.1 \\
\hline SDHA L349R fs*11 & GIST_24 & 1 & 1 & 1 \\
\hline SDHA c.457-3_457-1 delCAG & GIST_234 & 1 & ' & 1 \\
\hline SDHB C101V fs*3 & GIST_311 & I & ' & I \\
\hline SDHC E144Q & GIST_281 & $5.3 \%$ & $5.3 \%$ & 60.4 \\
\hline
\end{tabular}

Figure 2 Mapping of the GIST-associated mutations and truncations detected in the different patients on the human mitochondrial succinate dehydrogenase (SDH). The structure of the four subunits of the SDH protein complex were computed as previously described, ${ }^{6}$ adopting as a template the homologous pig counterpart (PDB code: 1ZOY). Color code of the subunits: SDH subunit A (SDHA) - yellow; SDH subunit B (SDHB) - fuchsia; SDH subunit C (SDHC) blue; SDH subunit D (SDHD) - green. The ball structures represent the amino-acid substitution positions: the blue for patient GIST_214, the red for patiets GIST_145 and GIST_10, and the yellow for patient GIST_281. The black stars indicate the location of premature truncations promoted by the three DNA deletions or the nonsense mutations. For each protein variations the distance from the protein flavin adenine dinucleotide (FAD)-binding site and the polar relative solvent accessibility area computed with the DSSP program ${ }^{14}$ are also listed. Relative solvent accessibility of each variation was computed both for the single subunit and for the complex. Different values of solvent accessibility indicate that the corresponding variation is located at the subunit interface. The character ' ${ }^{\prime}$ ' indicate previously reported mutations. ${ }^{6}$

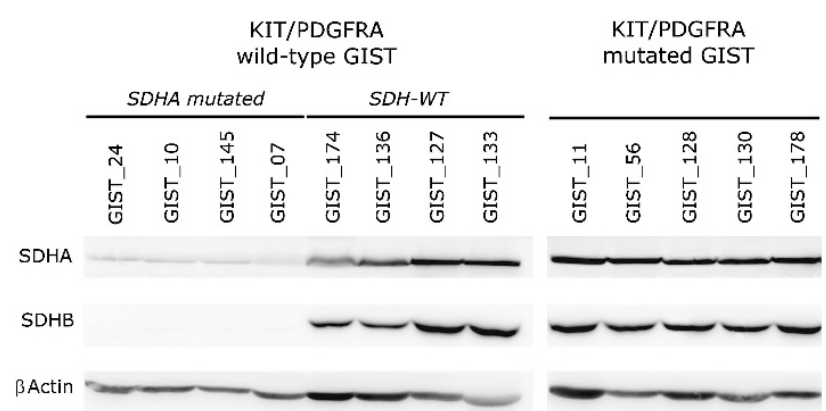

Figure 3 Western blot evaluation of SDHA and SDHB proteins. KIT/PDGFRA wild-type GIST harboring SDHA mutations have shown a remarkable inhibition of both SDHA and SDHB proteins with respect to KIT/PDGFRA wild-type GIST lacking SDH-mutations and to KIT/PDGFRA-mutated GISTs. $\beta$-Actin was used as a loading control.
SDHA mutation was identified only in the tumor; in another case, the SDHB mutation was found only in PB; in the remaining two cases, SDHB and SDHC mutations were identified in the tumor.

Germline mutations in SDHB, SDHC, and SDHD were seen in patients with the Carney-Stratakis syndrome, who are predisposed to developing paraganglioma and GIST. ${ }^{25-27}$ More recently, somatic mutations in SDH complex were found also in KIT/PDGFRA wildtype GIST patients who, apparently, did not have personal or familial history of paraganglioma and GIST. ${ }^{5-12}$ However, the presence of germline mutations seen in our series is interesting and should not be underestimated. At this time, it cannot be excluded that in this subset of adult KIT/PDGFRA wild-type GIST patients, without personal and family history of tumors but harboring germline mutations in SDH complex, the GIST may represent the first neoplastic event in a context of an attenuated form of CarneyStratakis syndrome or of a syndrome not yet clearly manifested and 

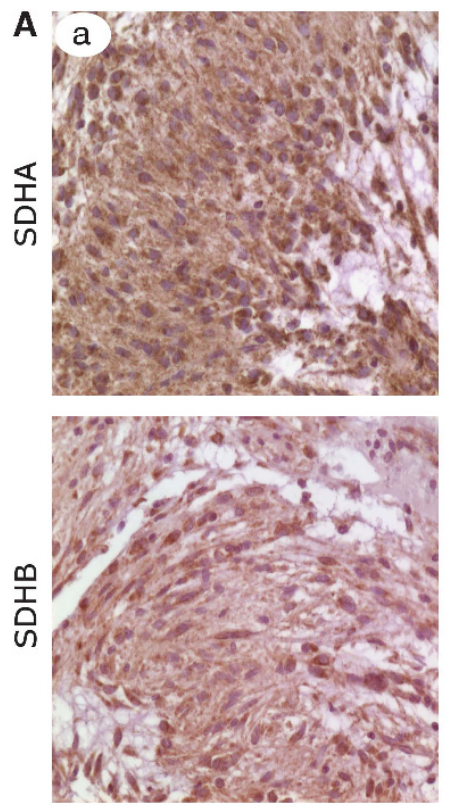

KIT/PDGFRA wild-type GIST (SDH-complex WT)
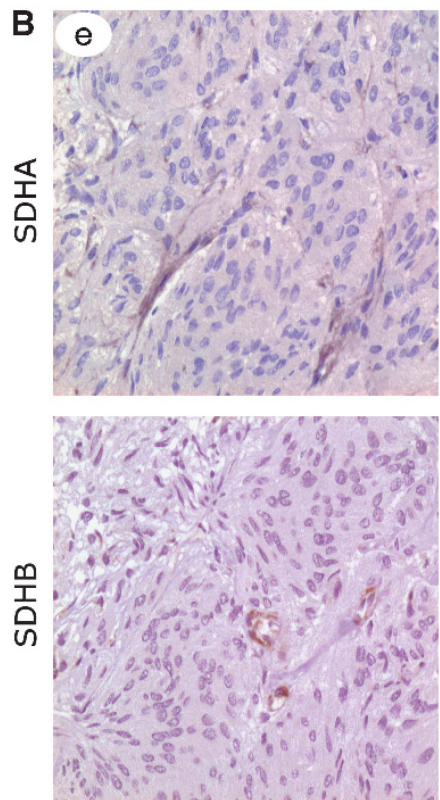

SDHA mutated
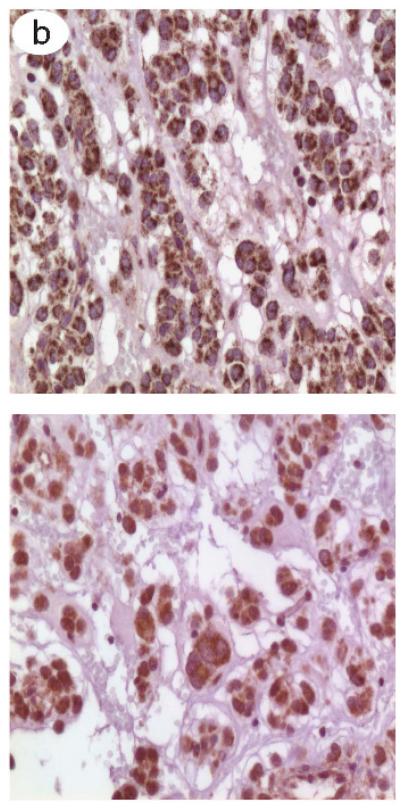

EX9 mutation
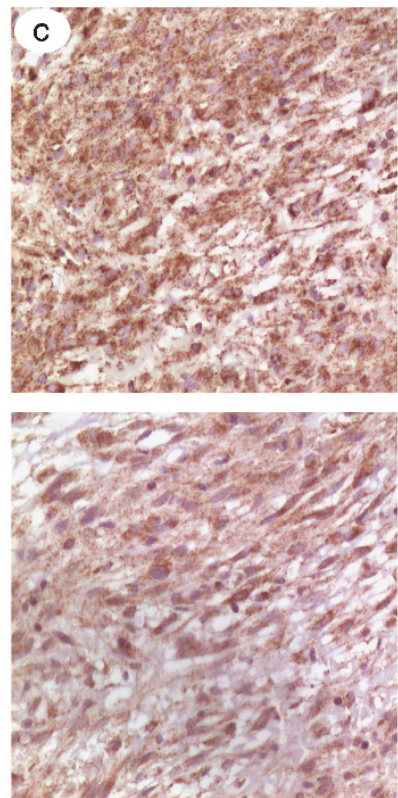

EX11 mutation
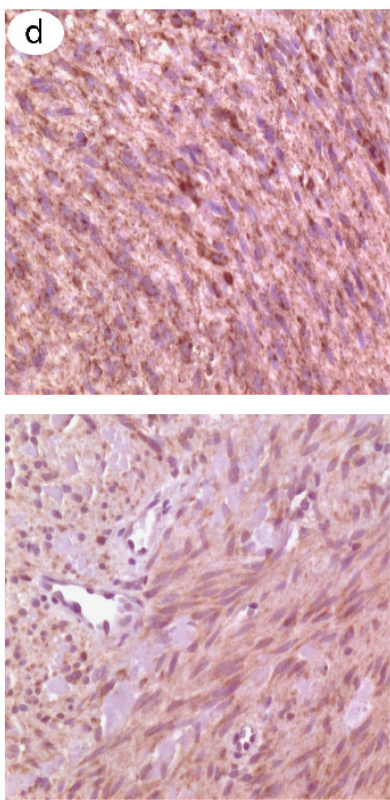

EX18 mutation
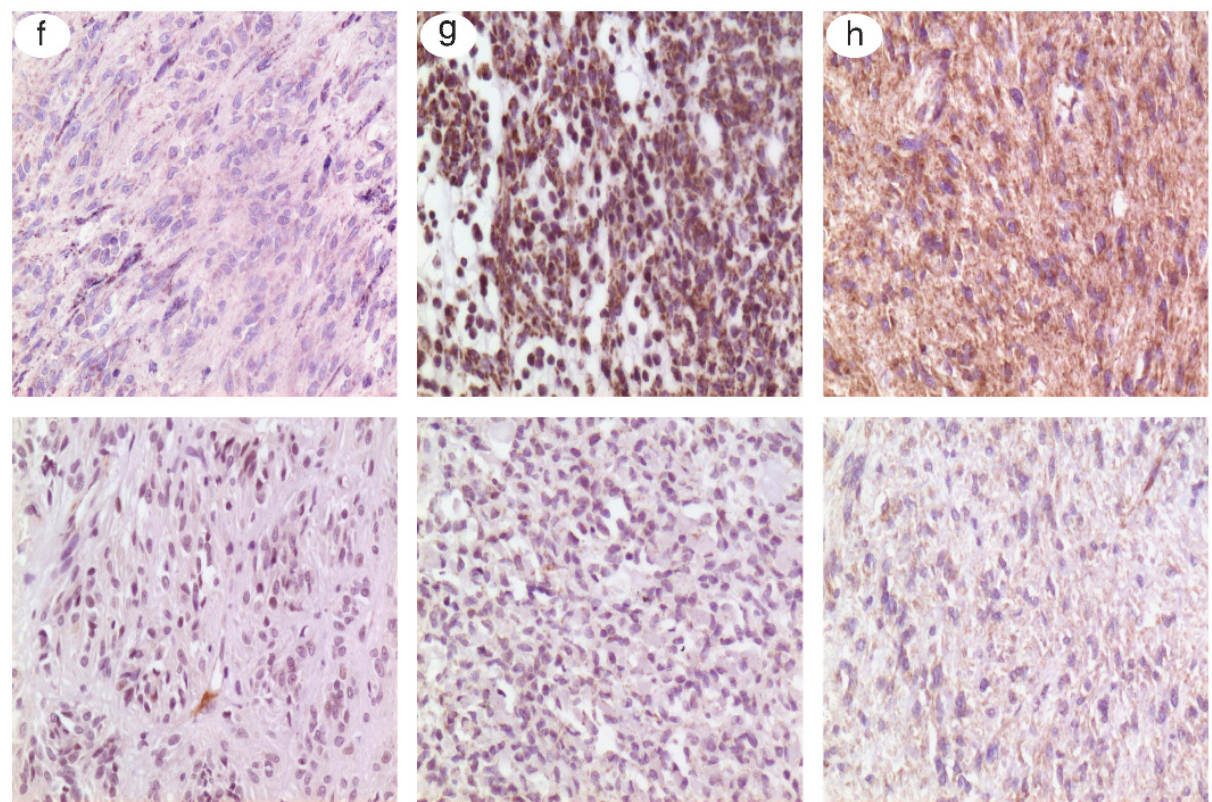

SDHA mutated

SDHB mutated

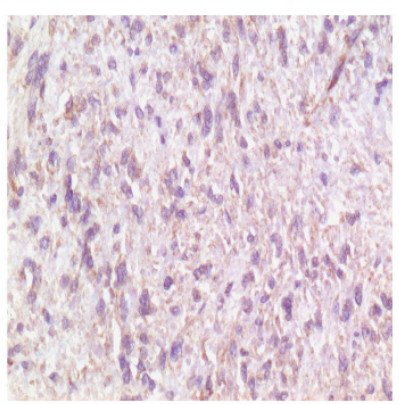

SDHC mutated

KIT/PDGFRA wild-type GIST

Figure 4 SDHA and SDHB immunohistochemistry. (A) Expression of SDHA and SDHB in mutated and KIT/PDGFRA wild-type GIST without SDH complex mutations. Strong granular staining in the cytoplasm and mitochondria of spindle and epithelioid tumor cells (a-d). (B) Expression of SDHA and SDHB in KIT/PDGFRA wild-type GIST with SDH complex mutations. Absence of SDHA and SDHB immunostaining in tumor cells (positive staining in blood vessels) in SDHA mutated patients (e, f); SDHA positive staining in tumor cells in SDHC/SDHB mutated patients (g, h).

defined. Data from long-term follow-up of these patients should be evaluated.

With regard to the proteins expressed, in our series, patients with KIT/PDGFRA wild-type GIST but mutated SDHA showed a negative staining for both SDHA and SDHB proteins, while patients with
SDHB or SDHC mutation showed a negative staining of SDHB (Figures 3 and 4). Therefore, loss of expression of SDHB protein is supported by mutations in all SDH subunits, ${ }^{4,5,7-12}$ while the loss of expression of SDHA is supported only by mutations of SDHA. Results of the SDHB IHC test suggest triaging the genetic testing in 
Table 3 SDHA and SDHB immunohistochemistry analysis performed on KIT/PDGFRA wild-type GIST

\begin{tabular}{|c|c|c|c|c|c|}
\hline \multirow{3}{*}{$\begin{array}{l}\text { ID } \\
\text { GIST_07a }\end{array}$} & \multicolumn{3}{|c|}{ SDH mutational status } & \multicolumn{2}{|c|}{$I H C$} \\
\hline & \multirow{2}{*}{$\begin{array}{l}\text { Subunits } \\
\text { SDHA }\end{array}$} & \multicolumn{2}{|c|}{ Mutation } & \multirow{2}{*}{$\begin{array}{c}\text { SDHA } \\
\text { Negative }^{b}\end{array}$} & \multirow{2}{*}{$\frac{S D H B}{\text { Negative }^{b}}$} \\
\hline & & c. $1151 \mathrm{C}>\mathrm{G}$ & p.S384X & & \\
\hline GIST_10 & SDHA & $\begin{array}{l}\text { c. } 91 \mathrm{C}>\mathrm{T} \\
\text { c. } 1765 \mathrm{C}>\mathrm{T}\end{array}$ & $\begin{array}{l}\text { p.R31X } \\
\text { p.R589w }\end{array}$ & Negative $^{b}$ & Negative $^{b}$ \\
\hline GIST_127 & & WT & WT & Positive $^{b}$ & Positive $^{b}$ \\
\hline GIST_133 & & WT & WT & Positive $^{\mathrm{b}}$ & Positive ${ }^{b}$ \\
\hline GIST_136 & & WT & WT & Positive ${ }^{b}$ & Positive $^{\mathrm{b}}$ \\
\hline GIST_145 & SDHA & $\begin{array}{l}\text { c. } 511 \mathrm{C}>\mathrm{T} \\
\text { c. } 1766 \mathrm{G}>\mathrm{A}\end{array}$ & $\begin{array}{l}\text { p.R171C } \\
\text { p.R589Q }\end{array}$ & Negative $^{\mathrm{b}}$ & Negative $^{b}$ \\
\hline GIST_174 & & WT & WT & Positive $^{b}$ & Positive $^{b}$ \\
\hline GIST_201 & & WT & WT & Positive & Positive \\
\hline GIST_202 & & WT & WT & Positive & Positive \\
\hline GIST_207 & & WT & WT & Positive & Positive \\
\hline GIST_219 & & WT & WT & Positive & Positive \\
\hline GIST_228 & & WT & WT & Positive & Positive \\
\hline GIST_234 & SDHA & c. $457-3 \_457$ & delCAG & Negative & Negative \\
\hline GIST_236 & & WT & WT & Positive & Positive \\
\hline GIST_241 & & WT & WT & ND & Positive \\
\hline GIST_270 & SDHB & c. $423+2$ & $\mathrm{~T}>\mathrm{A}$ & Positive & Negative \\
\hline GIST_275 & & WT & WT & Positive & ND \\
\hline GIST_276 & & WT & WT & Positive & Positive \\
\hline GIST_279 & & WT & WT & Positive & Positive \\
\hline GIST_281 & $\mathrm{SDHC}$ & c. $455 \mathrm{G}>\mathrm{C}$ & p.E144Q & Positive & Negative \\
\hline
\end{tabular}

Abbreviation: ND, not detected.

aThese patients were already described.

bData confirmed also by WB analysis.

SDHB, SDHC, and SDHD genes in familial pheochromocytoma/ paraganglioma; ${ }^{28}$ as well, this may also be useful in adult nonsyndromic KIT/PDGFRA wild-type GIST with same purpose, but, we suggest adding the subunit $\mathrm{A}$ to the list of genetic tests since our patients with SDHA mutations were SDHB negative and the mutations in SDHA were prevalent.

From a clinical point of view, during recent years some attempts to classify or to define specific characteristics of these groups of patients have been reported. Firstly, Rege et al ${ }^{29}$ suggested a new disease entity called 'pediatric type' for a small group of adult patients with KIT/ PDGFRA wild-type GIST with the same histological and clinical features as pediatric GIST. The tumor primarily arose from the stomach with a mean size of $5.4 \mathrm{~cm}$, with a predominant mixed epithelioid and spindle-cell morphology with a multinodular architecture, and mainly affected women with mean age of 31.5 years. Clinically, tumors often gave rise to lymph node metastases, both at the time of diagnosis or at sites of distant recurrence. None of the patients showed a radiologic response to imatinib, two patients showed a response to sunitinib, and most of them experienced an indolent clinical course. No other molecular defects potentially responsible for tumor development have been investigated in this type of disease. Of note, four of our six patients with SDHA mutations presented with pediatric-type characteristics (predominantly female, young adult age, primary GIST localized in the stomach, morphology of mixed spindle and epithelioid cells prevalent, and a metastatic GIST at diagnosis with an indolent course). Gill et a ${ }^{30,31}$ also discussed pediatric-type GIST in adults, although these authors did not agree with this nomenclature or definition, instead using the term 'type 2 GIST', or SDHB-protein expression negative GIST to describe a GIST population having the same morphologic, pathologic, KIT/PDGFRA genotype status, and clinical features. In fact, it has been previously proposed to divide GIST into two distinct types (1 and 2) only on the basis of the positive and negative immunostaining of SDHB protein, respectively. ${ }^{31}$ Then, Miettinen et $a^{30,31}$ reported the clinicopathologic, immunohistochemical, and molecular study of 66 gastric GIST who were found to be SDHB negative at IHC and called 'SDH-deficient GIST.'32 It was confirmed that all of these patients presented a KIT/PDGFRA wild-type genotype and mostly presented a disease with the same characteristics to the GIST population reported by Rege and Gill. No mutations in $\mathrm{SDH}$ subunits $\mathrm{B}, \mathrm{C}$, and $\mathrm{D}$ were found in these series but the subunit $\mathrm{A}$ was not studied. So presently, from a clinical point of view, the terms 'SDHB negative' or generally 'SDH-deficient' GIST describe a population of patients with GIST belonging to cancer syndromes such as Carney-Triad and Carney-Stratakis syndrome, (as opposed to neurofibromatosis type $1^{33}$ ) and to a subset of adult KIT/PDGFRA wild-type non-syndromic GIST mostly, but not all, with pediatrictype characteristics.

Mutations in SDH complex result in dysfunction of complex II of the electron transport chain in mitochondria and, consequently, in defective oxidative phosphorylation, which mediates a pseudohypoxic response. SDH dysfunction may have a role in the pathogenesis of a subtype of KIT/PDGFRA wild-type GIST, however, currently, the exact role of $\mathrm{SDH}$ mutations in carcinogenesis is not completely known. Recently, the hypothesis that it may function as a tumor suppressor gene was reported by Burnichon et $a^{34}$ who identified a germline SDHA mutation, p.Arg589Trp, associated with LOH in tumor in a woman affected by a catecholamine-secreting abdominal paraganglioma. The function of the mutated SDHA was assessed in vivo and in vitro, and it was found that mutated protein caused a loss of SDH enzymatic activity in tumor tissue and in a yeast model system. The authors also demonstrated, using IHC and transcriptomic studies, that the SDHA mutation caused pseudohypoxia, as with other $\mathrm{SDH}$ gene mutations, and may promote angiogenesis and cell proliferation. So the loss of SDH function may play a role in the pathogenesis of KIT/PDGFRA wild-type GIST and of paraganglioma through similar molecular pathways as seen in renal cell cancers that display loss of von Hippel-Lindau tumor suppressor function. ${ }^{35}$

Recently, the correlation between the overexpression of the insulinlike growth factor receptor 1 (IGF1R) protein and the status of SDH complex deficiency in KIT/PDGFRA wild-type GIST has been described suggesting that the IGF1R overexpression in this subset of patients may be driven by the loss of function of the SDH complex. ${ }^{36-39}$

Finally, we would like to emphasize that, currently, the majority of KIT/PDGFRA wild-type GIST do not harbor mutations in SDH complex or do not present SDH deficiency, so their molecular background is still unknown. The discovery of the oncogenetic event in this GIST population still represents a great challenge.

In conclusion, we report that about $27 \%$ of KIT/PDGFRA wildtype adult patients with apparently non-syndromic GIST have mutations in SDH genes with predominance for the subunit A. Patients with KIT/PDGFRA wild-type GIST SDHB negative at IHC should be screened for germline or de novo mutations at least in SDHB and SDHA genes. Moreover, these patients should be followed up for the risk of development of other cancers and recognized for appropriate management. 


\section{CONFLICT OF INTEREST}

The authors declare no conflict of interest.

\section{ACKNOWLEDGEMENTS}

This work was supported by Novartis Oncology, Origgio, Italy and by grants from Fondazione Vanini-Cavagnino, Bologna, Italy. The supporters did not participate in the design of the study, collection and analysis of data, or the decision to publish. Special thanks to the GIST Study Group members, University of Bologna, Bologna, Italy: Annalisa Altimari, Fausto Catena, Paolo Castellucci, Massimo Del Gaudio, Monica Di Battista, Valerio Di Scioscio, Giorgio Ercolani, Stefano Fanti, Pietro Fusaroli, Lidia Gatto, Walter Franco Grigioni, Elisa Gruppioni, Cristian Lolli, Alessandra Maleddu, Anna Mandrioli, Pier-Luigi Martelli, Maria Caterina Pallotti, Antonio Daniele Pinna, Paola Tomassetti, Maurizio Zompatori.

1 Maleddu A, Pantaleo MA, Nannini M, Biasco G: The role of mutational analysis of KIT and PDGFRA in gastrointestinal stromal tumors in a clinical setting. $J$ Trans/ Med 2011; 9: 75 .

2 Bajor J: Gastrointestinal stromal tumors in patients with type 1 neurofibromatois. Clin Exp Med J 2009; 3: 247-254.

3 Stratakis CA, Carney JA: The triad of paragangliomas, gastric stromal tumors, and pulmonary chondromas (Carney triad), and the dyad of paragangliomas and gastric stromal sarcomas (Carney-Stratakis syndrome): a molecular genetics and clinical implications. J Internal Med 2009; 266: 43-52.

4 Agaram NP, Wong GC, Guo T et al: Novel V600E BRAF mutations in imatinib-naive and imatinib-resistant gastrointestinal stromal tumors. Genes Chromosomes Cancer 2008; 47: 853-859.

5 Janeway KA, Kim SY, Lodish M et al: Defects in succinate dehydrogenase in gastrointestinal stromal tumors lacking KIT and PDGFRA mutations. Proc Natl Acad Sci USA 2011; 108: 314-318.

6 Pantaleo MA, Astolfi A, Indio V et al: SDHA loss-of-function mutations in KIT-PDGFRA wild-type gastrointestinal stromal tumors identified by massively parallel sequencing. J Natl Cancer Inst 2011; 103: 983-987.

7 Pantaleo MA, Nannini M, Astolfi A, Biasco GGIST Study Group Bologna. A distinct pediatric-type gastrointestinal stromal tumor in adults: potential role of succinate dehydrogenase subunit A mutations. Am J Surg Pathol 2011; 35: 1750-1752.

8 Italiano A, Chen CL, Sung YS et al: SDHA loss of function mutations in a subset of young adult wild-type gastrointestinal stromal tumors. BMC Cancer 2012; 12: 408

9 Wagner AJ, Remillard SP, Zhang YX, Doyle LA, George S, Hornick JL: Loss of expression of SDHA predicts SDHA mutations in gastrointestinal stromal tumors. Mod Pathol 2012; 26: 289-294.

10 Dwight T, Benn DE, Clarkson A et al: Loss of SDHA expression identifies SDHA mutations in succinate dehydrogenase-deficient gastrointestinal stromal tumor. $\mathrm{Am} J$ Surg Pathol 2012; 37: 226-233.

11 Celestino R, Lima J, Faustino A et al: Molecular alterations and expression of succinate dehydrogenase complex in wild-type KIT/PDGFRA/BRAF gastrointestinal stromal tumors. Eur J Hum Genet 2013; 21: 503-510.

12 Oudijk L, Gaal J, Korpershoek E et al: SDHA mutations in adult and pediatric wild-type gastrointestinal stromal tumors. Mod Pathol 2012; 26: 456-463.

13 Calabrese R, Capriotti E, Fariselli $\mathrm{P}$ et al: Functional annotations improve the predictive score of human disease-related mutations in proteins. Hum Mutat 2009; 30: $1237-1244$

14 Kumar P, Henikoff S, Ng PC: Predicting the effects of coding non-synonymous variants on protein function using the SIFT algorithm. Nat Proto 2009; 4: 1073-1081.

15 Adzhubei IA, Schmidt S, Peshkin L et al: A method and server for predicting damaging missense mutations. Nat Methods 2010; 7: 248-249.
16 Capriotti E, Fariselli P, Casadio R: I-Mutant2.0: predicting stability changes upon mutation from the protein sequence or structure. Nucleic Acids Res 2010; 33: W306-W310.

$17 \mathrm{Ng}$ PC, Henikoff S: SIFT: predicting amino-acid changes that affect protein function. Nucleic Acids Res 2003; 31: 3812-3814.

18 Pierleoni A, Indio V, Savojardo C et al: MemPype: a pipeline for the annotation of eukaryotic membrane proteins. Nucl Acids Res 2011; 39: 1-6.

19 Pettersen EF, Goddard TD, Huang CC et al: UCSF Chimera-a visualization system for exploratory research and analysis. J Comput Chem 2004; 25: 1605-1612.

20 Kabsch W, Sander C: Dictionary of protein secondary structure: pattern recognition of hydrogen-bonded and geometrical features. Biopolymers 1983; 22: 2577-2637.

21 Wallace AC, Laskowski RA, Thornton JM: LIGPLOT: a program to generate schematic diagrams of protein-ligand interactions. Protein Eng 1996; 8: 127-134.

22 Wang $M$, Marin A: Characterization and prediction of alternative splice sites. Gene 2006; 366: 219-227

23 Brunak S, Engelbrecht J, Knudsen S: Prediction of human MRNA donor and acceptor sites from the DNA sequence. J Mol Biol 1991; 220: 49-65.

24 Pantaleo MA, Nannini M, Saponara M et al: Impressive long-term disease stabilization by nilotinib in two pretreated patients with KIT/PDGFRA wild-type metastatic gastrointestinal stromal tumours. Anticancer Drugs 2012; 23: 567-572.

25 McWhinney SR, Pasini B, Stratakis CA: International Carney Triad and CarneyStratakis Syndrome Consortium: familial gastrointestinal stromal tumors and germline mutations. N Engl J Med 2007; 357: 1054-1056.

26 Pasini B, McWhinney SR, Bei T et al: Clinical and molecular genetics of patients with the Carney-Stratakis syndrome and germline mutations of the genes coding for the succinate dehydrogenase subunits SDHB, SDHC, and SDHD. Eur J Hum Genet 2008; 16: 79-88.

27 Pasini B, Stratakis CA: SDH mutations in tumorigenesis and inherited endocrine tumours: lesson from the phaeochromocytoma-paraganglioma syndromes. J Int Med 2009; 266: 19-42.

28 Gill AJ, Benn DE, Chou A et al: Immunohistochemistry for SDHB triages genetic of SDHB, SDHC and SDHD in paraganglioma-pheocromocytoma syndromes. Hum Pathol 2010; 41: 805-814.

29 Rege TA, Wagner AJ, Corless CL et al: 'Pediatric-type' gastrointestinal stromal tumors in adults: distinctive histology predicts genotype and clinical behaviour. Am J Surg Pathol 2011; 35: 495-504.

30 Gill AJ, Chou A, Vilain RE, Clifton-Bligh RJ: 'Pediatric-type' gastrointestinal stromal tumors are SDHB negative ('type 2') GISTs. Am J Surg Pathol 2011 : 35: 1245-1247.

31 Gill AJ, Chou A, Vilain R et al: Immunohistochemistry for SDHB divides gastrointestinal stromal tumors (GISTs) into 2 distinct types. Am J Surg Pathol 2010; 34: 636-644.

32 Miettinen M, Wang Z-F, Sarlomo.Rikala M et al: Succinate dehydrogenase-deficient GISTs: a clinicopathologic, immunohistochemical, and molecular genetic study of 66 gastric GISTs with prediction to young age. Am J Surg Pathol 2011; 35: $1712-1721$.

33 Wang JH, Lasota J, Miettinen M: Succinate dehydrogenase subunit B (SDHB) is expressed in neurofibromatosis 1-associated gastrointestinal stromal tumors (Gists): implications for the SDHB expression based classification of Gists. J Cancer 2011; 2 : 90-93.

34 Burnichon N, Brière JJ, Libé R et al: SDHA is a tumor suppressor gene causing paraganglioma. Hum Mol Genet 2010; 19: 3011-3020.

35 Linehan WM, Srinivasan R, Schmidt LS: The genetic basis of kidney cancer: a metabolic disease. Nat Rev Urol 2010; 7: 277-285.

36 Nannini M, Astolfi A, Paterini $P$ et al: Expression of insulin growth factor receptor 1 (IGF1R) in KIT/PDGFRA wild-type GIST with succinate dehydrogenase complex dysfunction. Future Oncol 2012; 9: 121-126.

37 Chou A, Chen J, Clarkson A et al: Succinate dehydrogenase-deficient GISTs are characterized by IGF1R overexpression. Mod Pathol 2012; 25: 1307-1313.

38 Belinsky MG, Rink L, Flieder DB et al: Overexpression of insulin-like growth factor 1 receptor and frequent mutational inactivation of SDHA in wild-type SDHB-negative gastrointestinal stromal tumors. Genes Chromosomes Cancer 2012; 52: 214-224.

39 Lasota J, Wang Z, Kim SY, Helman L, Miettinen M: Expression of the receptor for type insulin-like growth factor (IGF1R) in gastrointestinal stromal tumors: an immunohistochemical study of 1078 cases with diagnostic and therapeutic implications. Am J Surg Pathol 2012; 37: 114-119.

Supplementary Information accompanies this paper on European Journal of Human Genetics website (http://www.nature.com/ejhg) 